\title{
Complete resection of a primitive neuroectodermal tumour arising in the bladder of a 31-year-old female after neoadjuvant chemotherapy
}

\author{
Cameron J. Lam; Bobby Shayegan, $M D^{2}$
}

'Michael G. DeGroote School of Medicine, McMaster University, Hamilton, ON, Canada; '2Division of Urology, McMaster University, Hamilton, ON, Canada

Cite as: Can Urol Assoc J 2016;10(7-8):E264-7. http://dx.doi.org/10.5489/cuaj.3808 Published online July 12, 2016

\section{Abstract}

Primitive neuroectodermal tumours (PNET) that arise in the urinary bladder are an extremely rare occurrence. Very few cases have been reported so far in the literature ${ }^{1-13}$ and we report another case here in a 31-year-old-female. The patient presented with polyuria, gross hematuria, followed by development of anuria, and was discovered to have a $9.4 \mathrm{~cm}$ mass arising in the posterolateral aspect of the bladder. Histologically, the tumour showed small, round, blue cells. Further analysis using break-apart fluorescent in situ hybridization (FISH) revealed non-random chromosomal translocations of the ews gene suggestive of Ewing sarcoma (ES)/ PNET. The patient completed seven cycles of neoadjuvant chemotherapy, which significantly reduced the size of the lesion. Due to the location of the lesion, surgical resection of the entire bladder and urethra with use of a continent cutaneous reservoir was performed. Here, the management of a 31-year-old female with ES/ PNET arising from the bladder is reported.

\section{Introduction}

Primitive neuroectodermal tumours (PNET) are classified as a member of the Ewing family of tumours (EFTs). PNET is classified into central nervous system (CNS) PNET and peripheral PNET, with peripheral PNET considered to be much less common. PNETs are phenotypically similar to Ewing sarcoma (ES), composed of small, round, blue cells that exhibit specific chromosomal translocations and are often positive for CD99 antibodies. These tumours have been more commonly identified in young adults and adolescents and are relatively aggressive. ${ }^{6}$ Primary PNETs arising in the bladder are extremely rare and few cases have been reported in the English literature to date. ${ }^{1-13}$ Here we present a case of a primary bladder PNET diagnosed in a 30-year old female.

\section{Case report}

In October 2014, a 30-year-old female presented with polyuria and gross hematuria. She was subsequently referred to our urology clinic in November 2014, following development of anuria and identification of a $6.4 \times 9.4 \times 7.7 \mathrm{~cm}$ bladder mass on ultrasound and transurethral resection of bladder tumour (TURBT). Otherwise, her previous medical and family history was unremarkable. A repeat TURBT with examination under anesthesia was performed, which identified a mobile tumour on the posterolateral aspect of the bladder not fixed to any adjacent organs.

Histopathological examination of specimens obtained during tumour resection revealed poorly differentiated malignant neoplasm with papillary features and small, round, blue cell tumour morphology. Tumour cells were positive for CD99 antibody, focal S100, and synaptophysin, and focally positive for desmin. A EWS-FLI-1 gene transcription was identified using break-apart fluorescent in situ hybridization (FISH) of tumour cells. These histological findings increased the suspicion of a diagnosis of a ES/PNET.

Followup magnetic resonance imaging (MRI) confirmed an $8.1 \mathrm{~cm}$ tumour filling most of the right side of the bladder, with transmural extension to the base and attachment without invasion to the right ovary and adnexa. Further staging did not show any evidence of metastases.

Neoadjuvant chemotherapy consisting of alternating VDC (vincristine, doxorubicin, cyclophosphamide) and IE (ifosfamide, etoposide) was started following diagnosis. Aside from a single episode of febrile neutropenia, which she recovered from uneventfully, our patient tolerated chemotherapy quite well. Following five cycles of chemotherapy, a multi-planar, multi-sequence MRI showed a markedly reduced tumour measuring $2.5 \times 2.5 \times 2.1 \mathrm{~cm}$, extending through the full thickness of the bladder with no extravesical extension or metastases.

The case was discussed at the multidisciplinary cancer conference and while the tumour is believed to be radi- 


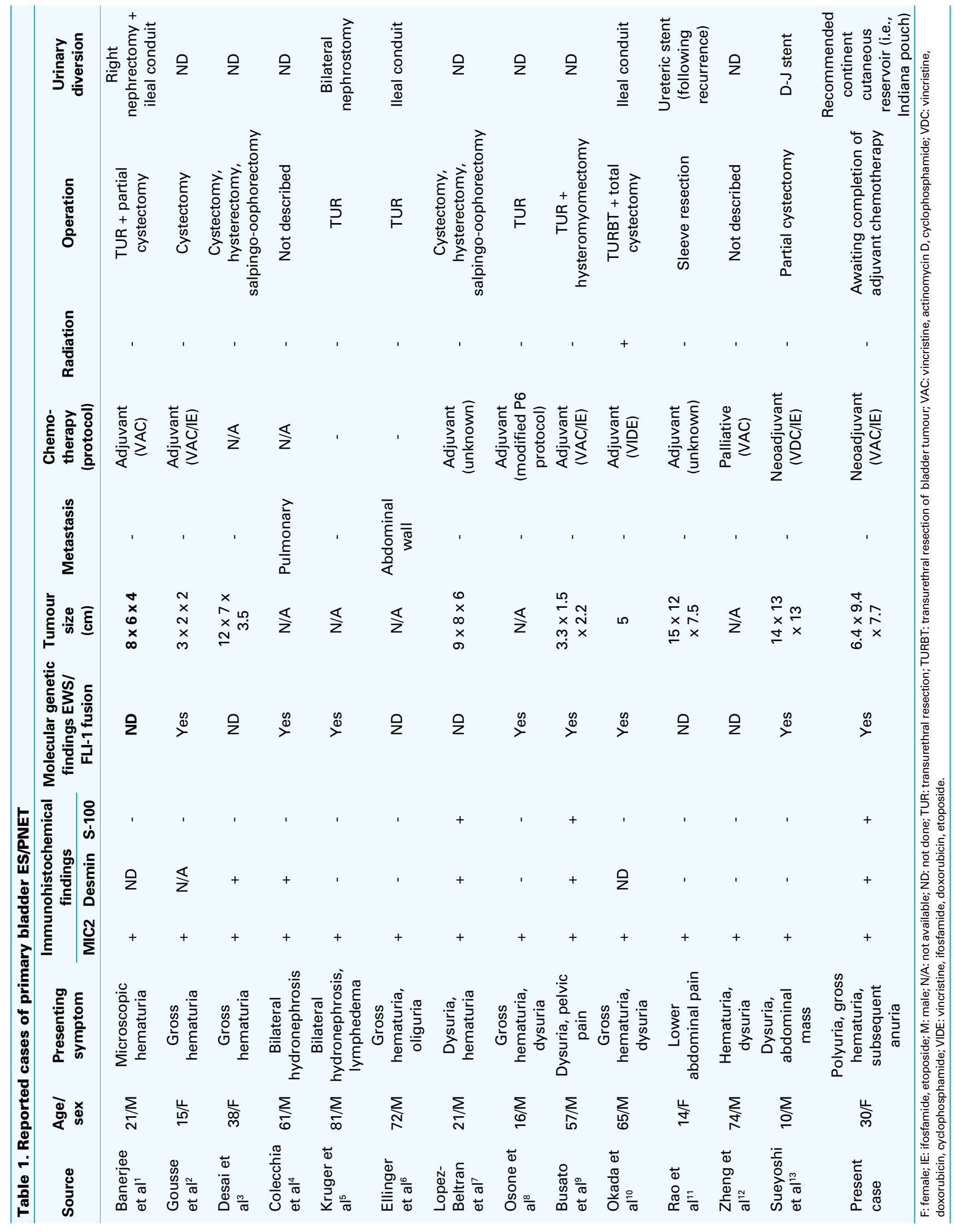


osensitive, given her very young age and the concern for secondary malignancy, a recommendation for surgical excision was made. Due to the location of the tumour adjacent to the urethra and bladder neck, an orthotopic neobladder was not advised.

Before surgical intervention, our patient received a total of seven cycles of neoadjuvant chemotherapy. Gynecologic organ-sparing radical cystourethrectomy with node dissection and urinary diversion by means of an Indiana pouch was performed in November 2015. Pathology following the procedure revealed a $\mathrm{pT} 1 \mathrm{bN} 0$ tumour of $3 \mathrm{~cm}$ in size and a negative surgical margin of $0.7 \mathrm{~cm}$. The histological type was classified as an extraskeletal ES with 54\% necrosis. The specimen was positive for CD99, CD56, and synaptopysin, and negative for CK7, CK20, and AE1/AE3 - confirming EWSR gene rearrangement and an ES. At the current stage, no further treatment will be required.

\section{Discussion}

Prior to this case report, there have been few other cases of primary ES/PNET in the bladder reported in the English literature (Table 1). Patients ranged in age at time of diagnosis from 10- 81 years, with a mean age of 41 years. The prognosis for adult cases of ES/PNET is generally considered to be worse than pediatric cases. ${ }^{14}$ In general, ES/PNET is considered to have more potential to develop in children, though 11 of the 13 reported cases of ES/PNET in the bladder were found in patients over the age of 20. Reported presenting symptoms have included dysuria, oliguria, anuria, polyuria, pelvic and abdominal pain, and hematuria.

These tumours are often highly aggressive and metastasize rapidly to the bone marrow, bone, and lung. Identification of these tumours is important to help guide the treatment strategy, as rapid therapy is required and the diagnosis of small blue round cell tumours is broad. A diagnosis of ES/ PNET requires a combination of histological, immunohistochemical, and molecular analysis of tumour cells. ${ }^{?}$

Histologically, PNETs of the bladder appear as poorly differentiated sheets of small, round cells that stain blue on hematoxylin and eosin (H\&E) staining. Several small, round, blue cell tumours of the bladder are indistinguishable by simple H\&E staining and require further diagnostic methods, as the prognosis and management are significantly affected. Non-random chromosomal translocations of the ews gene on chromosomes $22 q 12$ and $11 q 24$ reveals clear evidence of ES/PNET and excludes other small, round, blue cell tumour pathology.

Treatment for PNET arising from the bladder was based on that used in ES treated as an aggressive form by use of adjuvant chemotherapy. ${ }^{15,16}$ In our present case, seven courses of systemic chemotherapy with VDC/IE, showed a significant reduction in the size of the tumour from $8.1 \mathrm{~cm}$ to $2.5 \mathrm{~cm}$. Radical resection of the bladder and urethra with continent diversion by Indiana pouch was completed following seven cycles of chemotherapy.

In our case, neoadjuvant chemotherapy was successful in reducing the size of a primitive neuroectodermal tumour arising from the bladder of a 31-year-old female. Surgical intervention followed chemotherapy and no further treatment will be required at this time. The patient will continue with regular monitoring and followup.

\section{Conclusion}

Our case demonstrates that complete surgical resection of the bladder and urethra following neoadjuvant chemotherapy without radiotherapy was a safe and effective for management of PNET in a young woman.

Competing interests: The authors declare no competing personal or financial interests.

This paper has been peer-reviewed.

\section{References}

1. Baneriee SS, Eyden BP, McVey RJ, et al. Primary peripheral primitive neuroectodermal tumour of urinary bladder. Histopathology 1997;30:486-90. http://dx.doi.org/10.1046/j.1365-2559.1997.00524.x

2. Gousse AE, Roth DR, Popek EJ, et al. Primary Ewing's sarcoma of the bladder associated with an elevated antinuclear antibody titer. J Urol 1997;158:2265-6. http://dx.doi.org/10.1016/S0022$5347(01) 68231-4$

3. Desai S. Primary primitive neuroectodermal tumour of the urinary bladder. Histopathology 1998;32:477-8. http://dx.doi.org/10.1046/j.1365-2559.1998.0358a.x

4. Colecchia M, Dagrada GP, Poliani PL, et al. Immunophenotypic and genotypic analysis of a case of primary peripheral primitive neuroectodermal tumour (PPNET) of the urinary bladder. Histopathology 2002;40:1089. http://dx.doi.org/10.1046/i.1365-2559.2002.1340e.x

5. Kruger S, Schmidt $H$, Kausch I, et al. Primitive neuroectodermal tumour (PNET) of the urinary bladder. Pathol Res Pract 2003;199:751-4. http://dx.doi.org/10.1078/0344-0338-00492

6. Ellinger J, Bastian PJ, Hauser $S$, et al. Primitive neuroectodermal tumour: Rare, highly aggressive differential diagnosis in urologic malignancies. Urology 2006;68:257-62. http://dx.doi.org/10.1016/i. urology.2006.02.037

7. Lopez-Beltran A, Perez-Seoane C, Montironi R , et al. Primary primitive neuroectodermal tumour of the urinary bladder: A clinico-pathological study emphasising immunohistochemical, utrastructural and molecular analyses. J Clin Pathol 2006;59:775-8. http://dx.doi.org/10.1136/icp.2005.029199

8. Osone $\mathrm{S}$, Hosoi $\mathrm{H}$, Tanaka $\mathrm{K}$, et al. A case of a ewing sarcoma family tumour in the urinary bladder after treatment for acute lymphoblastic leukemia. J Pediatr Hematol Oncol 2007;29:841-4. http://dx.doi. org/10.1097/MPH.0b013e318158155b

9. Busato WF Jr, Almeida GL, Ogata DC. Primary primitive neuroectodermal tumour of the bladder: Histologic and clinical features of nine cases. Clin Genitourin Cancer 2011;9:63-7. http://dx.doi.org/10.1016/i. clgc.2011.03.002

10. Okada Y, Kamata S, Akashi T, et al. Primitive neuroectodermal tumour/Ewing's sarcoma of the urinary bladder: A case report and its molecular diagnosis. Int I Clin Oncol 2011;16:435-8. http://dx.doi. org/10.1007/s10147-010-0144-8

11. Rao RN, Sinha S, Babu $S$, et al. Fine-needle aspiration cytology of primitive neuroectodermal tumour of the urinary bladder: A case report. Diagn Cytopathol 2011;39:924-6. http://dx.doi.org/10.1002/dc.21585

12. Zheng $Y$, Tan $F$, Wang $L$, et al. Primary primitive neuroectodermal tumour of the urinary bladder: $A$ case report and literature review. Med Oncol 2011;28 Suppl 1:S388-91. http://dx.doi.org/10.1007/ s12032-010-9680-3 
PNET in the urinary bladder

13. Sueyoshi R, Okawada M, Füjimura J, et al. Successful complete resection of Ewing sarcoma arising from the bladder in a 10-year-old boy after chemotherapy. Pediatr Surg Int 2014;30:965-9. http://dx.doi. org/10.1007/s00383-014-3573-z

14. de AlavaE, Kawai A, Healey JH, et al. EWS-FLII fusion transcript structure is an independent determinant of prognosis in Ewing's sarcoma. J Clin Oncol 1998;16:1248-55.

15. Skubitz KM, D'Adamo DR. Sarcoma. Mayo Clin Proc 2007;82:1409-32. http://dx.doi. org/10.4065/82.11.1409
16. Grier HE, Krailo MD, Tarbell NJ, et al. Addition of ifosfamide and etoposide to standard chemotherapy for Ewing's sarcoma and primitive neuroectodermal tumour of bone. N Engl J Med 2003;348:694-701. http://dx.doi.org/10.1056/NEJMoa020890

Correspondence: Dr. Bobby Shayegan, Division of Urology, McMaster University, Hamilton, ON, Canada; shayeb@mcmaster.ca 